\title{
APLICACIÓN DE LOS MODELOS DE SIMULACIÓN EN ENTORNOS PRODUCTIVOS BAJO LA METODOLOGÍA DE TEORÍA DE LAS RESTRICCIONES
}

\author{
Application of simulation models based on the theory of constraints in production \\ environments
}

\author{
Julián Alberto Uribe-Gómez \\ MSc en Gestión Tecnológica, Facultad de Ciencias Económicas y Administrativas, Instituto Tecnológico \\ Metropolitano ITM, Medellín-Colombia, julianuribe@itm.edu.co \\ Santiago Quintero-Ramírez \\ PhD en Ingeniería Industria y Organizaciones, Grupo de Investigación en Gestión de la Tecnología y la \\ Innovación, Escuela de Ingenierías, Universidad Pontificia Bolivariana, Medellín-Colombia, \\ santiago.quintero@upb.edu.co
}

\section{Cómo citar / How to cite}

Uribe-Gómez, J. A. y Quintero-Ramírez, S. (2017). Aplicación de los modelos de simulación en entornos productivos bajo la metodología de teorías de las restricciones. Revista CEA, 3(6), 11-27.

Recibido: 16 de enero de 2017

Aceptado: 30 de marzo de 2017

\section{Resumen}

La Teoría de restricciones permite identificar dificultades en los procesos productivos a través de lo que se ha denominado cuellos de botella, y bajo una perspectiva rigurosa y sistemática busca la solución óptima para lograr el objetivo empresarial, el cual es generar dinero. El objetivo primordial de este trabajo fue evidenciar cómo el proceso sistemático de la teoría de restricciones, puede ser replicado de manera simple bajo la perspectiva de la modelación y simulación de los comportamientos que circunscriben dicha teoría. La simulación, en este caso, se utiliza como una metodología para ejemplificar y demostrar el pensamiento contra intuitivo de esta teoría, que emerge debido a que las empresas aún consideran los inventarios y su acumulación, como parte fundamental para la generación de beneficios en las organizaciones. En términos generales, con el modelo de simulación se logró demostrar que, reduciendo los inventarios en proceso de una cadena productiva, el comportamiento de los gastos operativos disminuye, generando así un mayor flujo de dinero a través de la empresa.

Palabras clave: simulación, modelos, software, metodología, manufactura, producción.

\begin{abstract}
The Theory of Constraints enables to identify difficulties, called bottlenecks, in production processes. It seeks to find an optimal solution from a thorough and systematic perspective to achieve the business objective, generating profit. This work aims to show the way the systematic process of the Theory of Constraints can be replicated in a simple way by means of modeling and simulating the behaviors that circumscribe this theory. In this case, simulation is the selected method to exemplify and demonstrate the counter-intuitive approach of this theory. The latter has been
\end{abstract}


described so because companies still consider inventories and accumulation to be fundamental parts of profit generation in the organizations.

In general terms, the simulation model demonstrated that downscaling inventories in a production chain reduces operating expenses, thus generating a greater cash flow in the company.

Keywords: simulation, models, software, method, manufacturing, production.

\section{INTRODUCCIÓN}

Durante muchos años, las empresas centraron su trabajo en el paradigma de la producción en masa, la acumulación de inventarios y materia prima para garantizar su permanencia a mediano y largo plazo, sin embargo, con los cambios actuales de consumo, las empresas e industrias, en general, buscan formas de incrementar sus servicios, como también sus ganancias, tratando de lograr sostenibilidad en el tiempo.

Han sido muchas las diferentes teorías industriales y productivas que han buscado responder o solucionar situaciones con respecto a la producción, buscando un equilibrio entre demanda-producción-oferta. Es así como, la búsqueda constante de dicho equilibrio empresarial ha llevado a que brote en las organizaciones la Teoría de las Restricciones (en adelante TOC por su sigla en inglés), cuya estrategia se basa en entender la estructura de un sistema productivo, viéndolo como una cadena y enfocándose en los eslabones más débiles, con miras a buscar el equilibrio que mejore el desempeño de la organización. Para ello las operaciones deben ser estabilizadas, requiriendo identificar y alterar las políticas contraproducentes
(Aguilera, 2000) que afectan el desempeño de la organización.

Esta teoría, y su metodología, ha llevado a expandirse a diversos ambientes, como lo son: la logística, la cadena de suministro, la gestión de proyectos, contabilidad, investigación y desarrollo, compra, ventas entre otros.

Algunas empresas que han aplicado con éxito la TOC, según la revista Fortune, son: 3M, Amazon, Boeing, Delta Airlines, Ford, Motor Company, General Electric y General Motors; estas empresas han alcanzado mejoras representativas a través de la implementación de esta metodología (Nanfang, Kaijun, y Tian, 2008).

Por lo tanto, es importante con esta teoría y su metodología, que las empresas conozcan a fondo la dinámica de su propia estructura en términos de procesos, a fin de sobrevivir en el tiempo frente a una competencia globalizada, así como a un ambiente turbulento en el cual se comportan los negocios actuales.

El presente trabajo ejemplifica y demuestra mediante la simulación, los beneficios de la TOC en el ámbito industrial, sin dejar de lado las ventajas inherentes que tiene la metodología de simulación para entender problemas complejos en el campo empresarial, adquiriendo los conocimientos y la experiencia global del funcionamiento de una organización.

Para tal fin, este trabajo se estructura así: el punto dos presenta una revisión conceptual de la Teoría de Restricciones; el punto tres se enfoca en la fundamentación teórica matemática de la TOC; en el cuarto punto se presenta la revisión conceptual acerca de la simulación; en el punto cinco, se presentan los resultados y la validación del modelo; las conclusiones y el trabajo futuro se presentan en el punto seis. 


\section{REVISIÓN CONCEPTUAL DE LA TEORÍA DE LAS RESTRICCIONES (TOC)}

La TOC desarrollada por los profesores Goldratt y Cox (1984) (Golmohammadi, 2015) representa una gran innovación en el ámbito de la producción. Goldratt sugiere que el principal propósito que tiene una organización, ahora y en el futuro, es hacer dinero y generar ganancias (Costas, Ponte, de la Fuente, Pino, y Puche, 2015) (Aguilera, 2000), esto quiere decir que la TOC se basa en la teoría de sistemas, siendo este un sistema teleológico, es decir, que tiene un objetivo o propósito. Para lograr esto, la metodología se basa en un proceso de mejora continua de cinco pasos, que busca administrar el sistema eliminando las dificultades para lograr dicho propósito (Golddratt, 1984) .

El principio fundamental de la TOC enfatiza la importancia de identificar y eliminar los cuellos de botella, en otras palabras, lo que se conoce como las restricciones, sin embargo la dificultad latente se encuentra en identificar estas restricciones (Izmailov, 2014) en la práctica productiva o manufacturera, por lo tanto son estas restricciones las que evitan o impiden a la empresa o al sistema alcanzar su máximo desempeño en términos de sus propósitos presentes y futuros (Şimşit, Günay, y Vayvay, 2014).

En general, la TOC se enfoca en la filosofía de mejora continua para superar estas restricciones, pero para ello requiere responder según Şimşit et al. (2014), las siguientes preguntas presentadas en la Tabla 1 , donde se muestra el objetivo de cada una de ellas y donde la metodología de la TOC se debe desarrollar para alcanzar el desempeño deseado.
A partir de esto, se deben identificar los tipos de restricciones que un sistema puede tener y a partir de esto tomar las acciones pertinentes mediante las preguntas antes presentadas, estas, según Murphy (1996), se clasifican en:

- Físicas: como recursos, demanda de mercado, entregas en las ventas.

- Administrativas o procedimentales: como incentivos, tipos de mediciones e indicadores, inventarios, actitudes hacia los empleados.

- Conductuales o de comportamiento: como hábitos o actitudes.

Algunos autores han comparado los diversos métodos de mejoramiento productivos, buscando las diversas ventajas y desventajas de cada uno de ellos, de esta manera hallar el enfoque adecuado para cada caso, así, en la Tabla 2, se presenta el comparativo entre dos metodologías y la TOC, sin lugar a dudas TOC tiene beneficios similares y podría agrupar a métodos como Six Sigma y Lean Manufacturing. Pero cabe resaltar que las metodologías son complementarias debido a sus diversas concepciones.

La complementariedad siempre ha llamado la atención de la administración y la alta gerencia, así se puede ver que el JIT (Just In Time) enfatiza en "no haga lo que no sea necesario», el TQM (Total Quality Management) enfatiza en «no es suficiente hacer correctamente las cosas, lo más importante en hacer las cosas» y la TOC insisten en que el resultado global es determinado por la continua optimización local (Aguilera, 2000). 
Tabla 1. Preguntas de la TOC y su Objetivo

Table 1. Questions and Objectives in TOC

\begin{tabular}{ll}
\hline Pregunta & Objetivo \\
\hline ¿Qué cambiar? & Identificar el problema clave \\
¿Hacia a dónde cambiar? & Desarrollar soluciones simples y prácticas \\
¿Cómo causar el cambio? & Implementar las soluciones \\
\hline
\end{tabular}

Fuente: adaptado de Kasemset (2011).

Tabla 2. Comparativo de diversos sistemas de mejoramiento productivos

Table 2. Comparison of different manufacturing improvement systems

\begin{tabular}{|c|c|c|c|}
\hline Factor & Six Sigma & Lean & TOC \\
\hline Teoría & Reducir la variación & Remover el desperdicio & Administrar restricciones \\
\hline Foco & Enfoque de problema & Enfoque de flujo & Restricciones de sistema \\
\hline \multirow{6}{*}{ Efecto } & Salida de proceso uniforme & Mejora en desempeño & $\begin{array}{l}\text { Énfasis en la velocidad y } \\
\text { volumen }\end{array}$ \\
\hline & 'Menor desperdicio & 'Reducción de la variación & 'Uso del sistema existente \\
\hline & $\begin{array}{l}\text { 'Incremento de la tasa de } \\
\text { Throughput }\end{array}$ & 'Tasa de salida uniforme & 'Independencia de proceso \\
\hline & 'Reducción de inventario & 'Reducción de inventario & $\begin{array}{l}\text { 'Reducción de Inventario y } \\
\text { desperdicio }\end{array}$ \\
\hline & 'Mejora en calidad & $\begin{array}{l}\text { 'Nuevo sistema de } \\
\text { contabilidad }\end{array}$ & $\begin{array}{l}\text { 'Contabilidad de costos por } \\
\text { Throughput }\end{array}$ \\
\hline & $\begin{array}{l}\text { 'Reducción de la fluctuación } \\
\text { de proceso }\end{array}$ & 'Mejora en la calidad & 'Mejoramiento en calidad \\
\hline Debilidad & $\begin{array}{l}\text { Ignora la interacción del } \\
\text { sistema }\end{array}$ & $\begin{array}{l}\text { Ignora la estadística o el } \\
\text { sistema de análisis }\end{array}$ & $\begin{array}{l}\text { Necesidades mínimas de } \\
\text { trabajadores }\end{array}$ \\
\hline
\end{tabular}

Fuente: Kasemset (2011).

\section{METOdOlOgÍA DE LA TEORÍA DE RESTRICCIONES}

La TOC, como una metodología de aplicación práctica, requiere procedimientos sólidos y conjeturas a demostrar. De este modo, se definirán las variables que esta teoría utiliza, así como el diagrama de flujo procedimental del método, dado que se evalúa paso a paso y requiere, por lo tanto, de un algoritmo que recorra el sistema en búsqueda de restricciones.
La metodología considera tres métricas operacionales importantes (Costas et al., 2015):

- Throughput o ganancia: tasa o velocidad en la con la cual el sistema genera dinero a través de las ventas, esta relación se puede ver en la ecuación (3) (González y Calvachi, 2013).

- Inventario: dinero invertido en comprar ítems, los cuales intentan ser vendidos; esta relación se puede ver en la ecuación (2) (Aguilera, 2000). 
- Gastos operativos: dinero gastado para convertir inventario en ganancia. Aquí se consideran los salarios directos, indirectos y administrativos, intereses bancarios, depreciaciones, entre otros, esta relación se puede ver en la ecuación (1) (Aguilera, 2000).

También se consideran tres métricas financieras:

- Retorno de la inversión: tiene como propósito dimensionar el esfuerzo necesario para el alcance de un determinado nivel de ganancia (Aguilera, 2000), también indica que tan bien está siendo utilizado el dinero puesto por los accionistas de la empresa (Gonzalez y Calvachi, 2013).

- Flujo de dinero: se trata de un indicador de equilibrio que se constituye en un condición necesaria para el buen funcionamiento de la empresa (Aguilera, 2000).
- Utilidad neta: esta es una medida de carácter absoluto, mide la cantidad de dinero que el sistema empresa está generando (Aguilera, 2000).

Existe también otra métrica implícita dentro del sistema, pero que no es menos importante definir; esta variable relaciona la métrica operativa y la métrica financiera, esta variable es la productividad, la cual es definida como todas las estrategias y acciones que llevan a la empresa más cerca de la meta, es decir, a ganar más dinero. Solo las acciones que acerca la empresa a su meta se pueden definir como productivas, esta relación se puede evidenciar en la ecuación (4) (González y Calvachi, 2013).

De acuerdo con la TOC, estas métricas están relacionadas y el desempeño del sistema se encuentra concentrado en mejorarlas. Así, en orden de cumplir con el objetivo organizacional, el cual es hacer dinero, el sistema debe incrementar su ganancia reduciendo su inventario y sus gastos operativos (Şimşit et al., 2014).

Una vez definidas las variables, se expresarán a continuación las relaciones matemáticas:

\section{Utilidad Neta $=$ Ganancia - Gastos operacionales (1) \\ Retorno de la inversión $=\frac{\text { Utilidad Neta }}{\text { Inventario }}(2)$ \\ Ganancia $=$ Ventas Totales - Costo totalmente variable (3) \\ Productividad $=\frac{\text { Ganancia }}{\text { Gastos operacionales }}$

Definidas las medidas operacionales y financieras, para evaluar el sistema y verificar que el objetivo de "ganar dinero» se esté cumpliendo, es importante revisar la metodología empleada por la TOC para buscar las restricciones en el sistema y corregir las inestabilidades. A continuación, se presentan los pasos procedimentales de la TOC en la Tabla 3. 
Tabla 3. Pasos procedimentales de la TOC Table 3. TOC Steps

\begin{tabular}{cl}
\hline Pasos & TOC \\
\hline 1 & Identificar restricciones \\
2 & Explotar la restricción \\
3 & Subordinar el sistema a la restricción \\
4 & Elevar la restricción \\
5 & Chequear nuevas restricciones \\
\hline & Fuente: tomado de Kasemset (2011).
\end{tabular}

Por lo tanto, la TOC se enfoca en dos aspectos (Zhang, 2009):

- Todo sistema tiene al menos una restricción que impide alcanzar su desempeño más alto.

- La existencia de restricciones o cuellos de botella representan oportunidades de mejora.

Con base en esto, la TOC busca las restricciones o los cuellos de botella, según esta teoría estos son los que impiden al sistema generar óptimos indicadores operativos y financieros de desempeño, en resumen, las salidas de la empresa están determinada por sus restricciones o cuellos de botella, esto indica que es importante evaluar iterativamente la capacidad del sistema y de sus cuellos de

Conociendo la metodología seguida por la TOC, es importante definir el primer paso de la teoría, la cual es identificar la restricción. Si se tiene en cuenta que la salida del sistema está determinada por el cuello de botella o la restricción, entonces es lógico pensar en términos de la capacidad del cuello de botella (Ray, Sarkar, y Sanyal, 2010), esto implica en que las soluciones se deben concentrar en manejar esta restricción. A esto se le llama capacidad del recurso de la restricción (Kasemset, 2011). botella. A continuación, la Fig. 1 presenta el diagrama de flujo del proceso metodológico de la TOC.

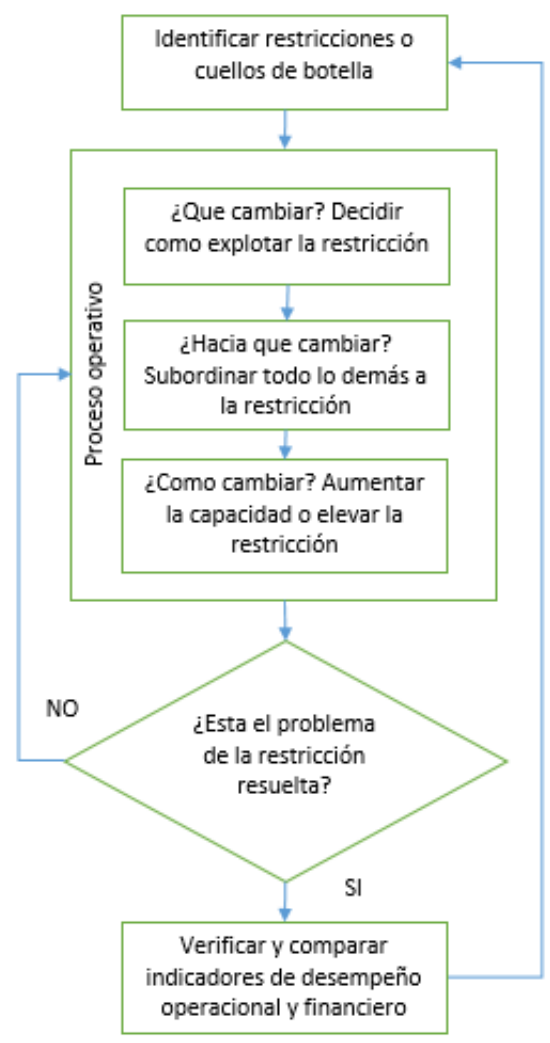

Figura 1. Diagrama de flujo proceso TOC Figure 1. TOC Flowchart Fuente: adaptado de Zhang (2009).

Principalmente, la revisión de literatura presenta dos maneras de evaluar las capacidades de producción, la primera evalúa un solo recurso y presenta un método de diferenciación entre la oferta y demanda de ese recurso, la segunda evalúa una sección con múltiples máquinas en múltiples productos y presenta una diferenciación similar, entonces para revelar un recurso con restricción se evalúa su capacidad, calculada a partir de la ecuación (6) menos su demanda, esta relación se ilustra en la ecuación (5) (Ray et al., 2010). 


$$
\text { Diferencia }=\sum t_{i j} D_{i}-\text { Capacidad }
$$

Donde:

$t_{i j}$ Es el tiempo de procesamiento

$D_{i}$ Demanda

$$
\begin{aligned}
\text { Capacidad = Días a la semana } & \\
& * \text { horas al día } \\
& * \text { minutos por hora }
\end{aligned}
$$

Para una sección con múltiples máquinas y múltiples productos, la ecuación propuesta para medir la capacidad de un recurso cuello de botella está definida por la ecuación (7) y (8) (Wu y Tsai, 2013), sin embargo, en general, la capacidad de un recurso está definida por la ecuación estándar (9).

$$
\begin{aligned}
& \text { Capacidad }=\sum L_{i}+\sum \frac{S_{i}\left[\frac{L}{e * h}\right]}{f_{i}} \\
& L_{i}=\frac{D_{i}}{P_{i}}
\end{aligned}
$$

$$
\text { Capacidad }=m * e * h
$$

Donde:

$L_{i}$ Es la capacidad requerida por producto i en un periodo.

$S_{i}$ Es el tiempo de preparación requerido para un producto i en un cuello de botella.

$e$ Días de trabajo por periodo.

$h$ Horas de trabajo por periodo.

$f_{i}$ Frecuencia de rotación.

$D_{i}$ Consumo promedio de producto en el periodo.

$P_{i}$ Salida de producción por hora por producto en el cuello de botella.

$m$ Número de máquinas.

\section{REVISIÓN CONCEPTUAL Y MODELO DE SIMULACIÓN}

La simulación es una técnica que permite crear modelos, apoyándose en programas informáticos, que posteriormente servirán para analizar el comportamiento de un sistema en diferentes circunstancias y generar destrezas en un ambiente controlado, analizando los posibles cambios y sus consecuencias. Desafortunadamente, aún son muchos los sectores industriales que no aprovechan de las ventajas que esta tecnología ofrece para la toma de decisiones, el ahorro de costes o la optimización de procesos industriales (Viveros y Chew, 2013).

Los paradigmas de simulación, entonces, tienen como objetivo comprender y modelar el comportamiento de entidades o variables en un sistema determinado. Esta ofrece formas fáciles para entender cómo los comportamientos afectan a otras variables del sistema (Macal y North, 2013), mediante tres elementos básicos: las variables, las relaciones o conexiones y el ambiente.

En términos generales, la simulación se enfoca en estudiar sistemas que contengan gran cantidad de objetos activos como lo son: personas, negocios o incluso proyectos, demanda, productos, etc., los cuales tengan asociados la variable tiempo y que tengan un comportamiento individual (Borshchev y Filippov, 2004).

Esto implica que la simulación es una herramienta muy útil para diseñar y analizar sistemas, donde los componentes interactúan bajo grados de dependencia y control (Humann y Madni, 2014; Akgün, Keskin, y Byrne, 2014). 


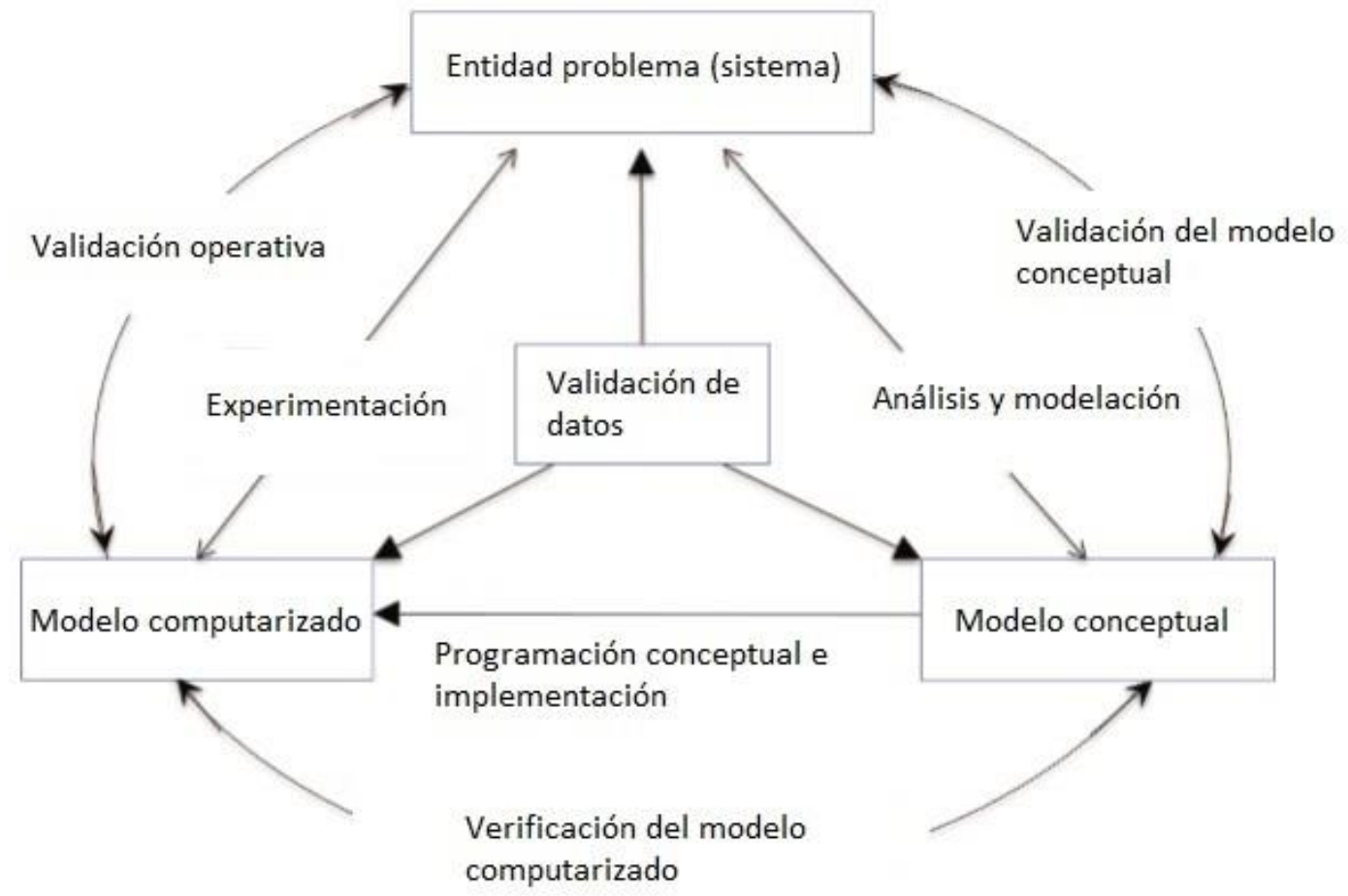

Figura 2. Metodología para la elaboración de modelo TOC

Figure 2. Methodology for the elaboration of TOC model

Fuente: adaptado de Sargent (2015).

\section{Propuesta de un modelo de simulación}

Para diseñar y desarrollar el modelo de simulación para la metodología de TOC, se utilizó la metodología propuesta por Quintero, Ruiz y Robledo (2017), la cual es presentada en la Fig. 2.

Desde esta perspectiva, el sistema a estudiar es la metodología TOC, el cual a través de la interacción de las tasas se afectan los factores que evitan que el sistema genere throughput, el modelo debe responder preguntas tales como: ¿Cuál es el problema que será investigado? ¿Cuáles son los supuestos más importantes para la creación del modelo? ¿Cuál es la lógica para la creación del modelo? De acuerdo con estas premisas, la Fig. 3 presenta un diagrama causal, donde se ejemplifican las relaciones, variables $y$ conceptos claves a trabajar en el simulador propuesto, con este, se desarrolla un diagrama de flujos y niveles presentado en la Fig. 4 con las siguientes características (Ortiz y Torres, 2008):

- Tres máquinas en línea, independientes y diferentes.

- Solo se procesa un tipo de pieza.

- La materia prima que entra en la máquina 1 continua su proceso hacia la maquina 2 y 3 , respectivamente.

- La materia prima llega tan pronto como se solicita.

- Todas las piezas terminadas son almacenadas en inventario y son vendidas determinadas por una probabilidad de venta.

- Existe una sola entrada y una sola salida del sistema. 
- No existen tiempos de transporte entre máquinas.

- Las máquinas no tienen tiempo de preparación debido a que se procesa un solo tipo de pieza.

- Cada máquina tiene un almacén de materia prima en proceso de capacidad infinita.

- Al iniciar la simulación, no se tiene materia prima en proceso, ni en inventario.

- El tiempo de la simulación es de 8 horas del turno de trabajo.

Las premisas o condiciones antes nombradas junto con las variables y el modelo de flujos y niveles se desarrollaron haciendo uso de la plataforma NetLogo versión 5.0.4 y el programa PowerSim, el modelo de simulación, comprende a su vez una interfaz de control del simulador de la TOC, esta se presenta en la Fig. 5.

Con el programa PowerSim se realizó el diagrama de causalidad, mientras que el programa NetLogo permitió ejecutar las simulaciones, particularmente este programa brinda agilidad y nivel de manipulación alto para desarrollar simulaciones de este tipo.

En la Fig. 5, se pueden apreciar las entradas más importantes del sistema, como son las tasas de producción, que es donde se centra principalmente la TOC, al querer sincronizar todos los flujos de trabajo; también se presentan las salidas más importantes, así como también sus respectivos gráficos de comportamiento.

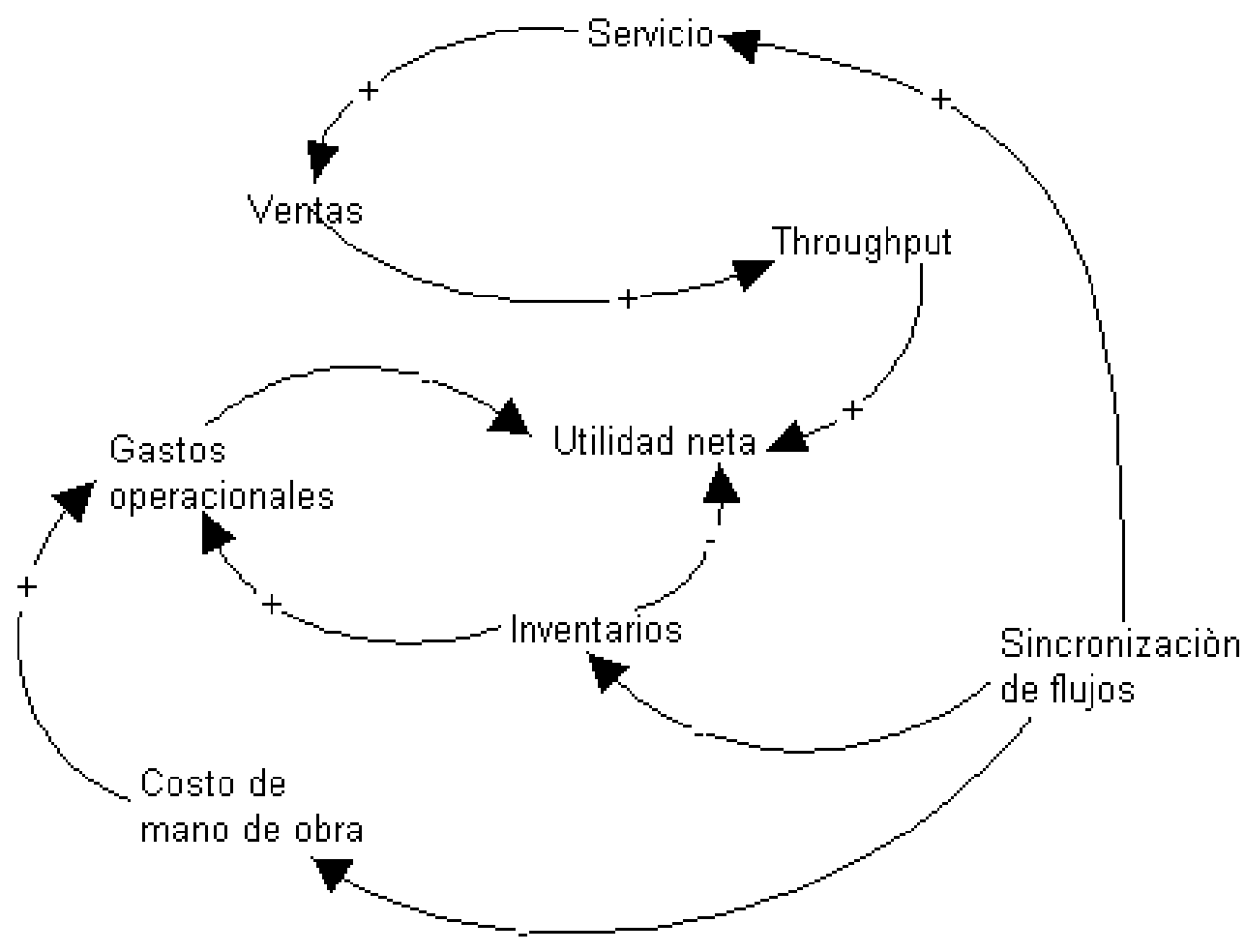

Figura 3. Diagrama causal de TOC

Figure 3. TOC Causal Loop Chart Fuente: adaptado de Marín y Gutiérrez (2013). 


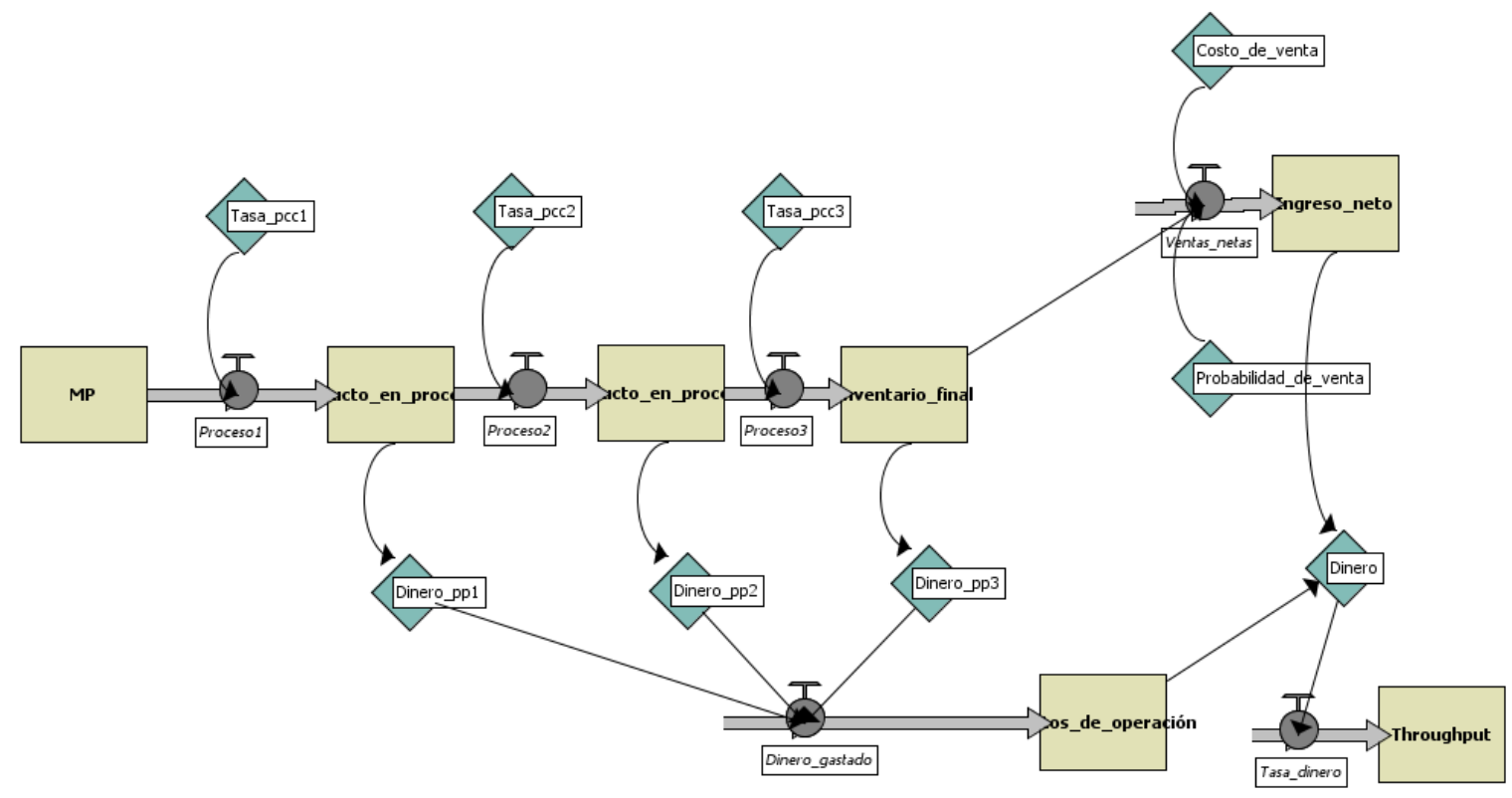

Figura 4. Diagrama de flujos y niveles

Figure 4. Stock and flows chart Fuente: elaboración propia.

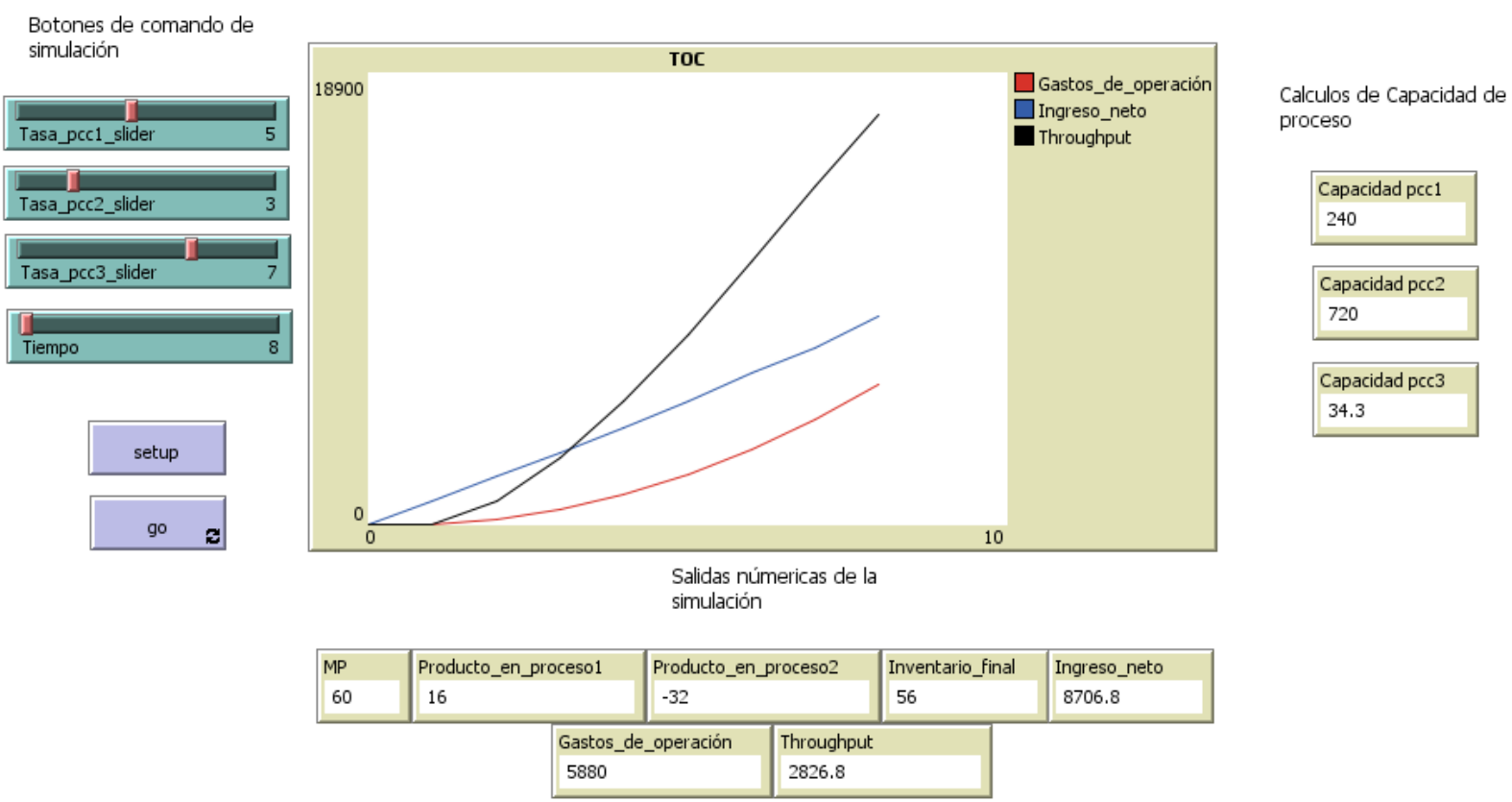

Figura 5. Interfaz modelo de simulación

Figure 5. Simulation Model Control

Fuente: elaboración propia. 


\section{RESULTADOS Y VALIDACIÓN DEL MODELO}

De acuerdo con la teoría presentada, lo que se busca con el modelo es evidenciar el comportamiento que TOC debe tener en el medio industrial, esto se desarrolló con un modelo de simulación bajo la plataforma NetLogo, la cual es un software basado en agentes, sin embargo, es utilizado aquí por su adaptabilidad.

Las entradas principales del simulador son las tasas de producción de cada estación de trabajo en el sistema; en un sistema convencional, cada estación de trabajo tiene un ritmo propio, debido a factores controlables y no controlables, por ejemplo, las maquinas que desarrollan la tarea y las personas. Cada estación de trabajo procesa el material e inmediatamente se almacena para ser recogido por la siguiente estación, hasta que finalmente cumple el ciclo y este material se almacena de nuevo como producto terminado.

La mano de obra, maquinaria, y almacenamiento de inventario corresponden a gastos de operación, por lo tanto, según la TOC, esto tendría que ser mínimo, sin embargo, la mano de obra ni la maquinaria pueden variar en este caso, ya que son costos fijos, así que se debe disminuir el inventario.

El producto terminado debe venderse para obtener ingresos, por lo tanto, esto debe ser maximizado, ya que el flujo de dinero, Throughput o ganancia según la TOC es la diferencia entre los ingresos menos los gastos.

De esta manera, se tienen dos escenarios: el primero presenta tres tasas distintas de producción de 5, 3 y 8 y el simulador calcula la capacidad de cada proceso, donde se selecciona el proceso con la mayor capacidad de producción, el cual es el cuello de botella o restricción respectivamente; este escenario base es habitual en muchos entornos productivos, los resultados son los presentados en la Fig. 6 y en la Tabla 4.

Los resultados muestran que tanto los gastos de operación como el ingreso neto crecen; mientras que el dinero o Throughput desciende al final de la simulación, por lo tanto, la tasa de generación de dinero no es la óptima.

El segundo escenario presenta la solución de la TOC, en este caso existe un pensamiento contra intuitivo en el abordaje del fenómeno, y es que según la TOC, la segunda tasa de producción es la menor, debido a que su capacidad es la más alta de las tres tasas de producción, por lo tanto es la restricción del sistema y el flujo de dinero se ve frenado, entonces si se subordina todo el sistema a esta tasa, es decir, las tasas de producción serán 3, 3 , y 3 respectivamente, los resultados del sistema son los presentados en la Fig.7 y la Tabla 5.

Para este escenario, los gastos de operación disminuyeron considerablemente, debido a que no existe inventario en proceso y los costos de almacenaje y reprocesos son nulos, por otra parte, bajo las mismas condiciones de venta el ingreso permanece creciendo, ahora el dinero o Throughput crece favoreciendo el flujo, este comportamiento es lo que predice la TOC para favorecer el objetivo organizacional, el cual es «hacer dinero» 


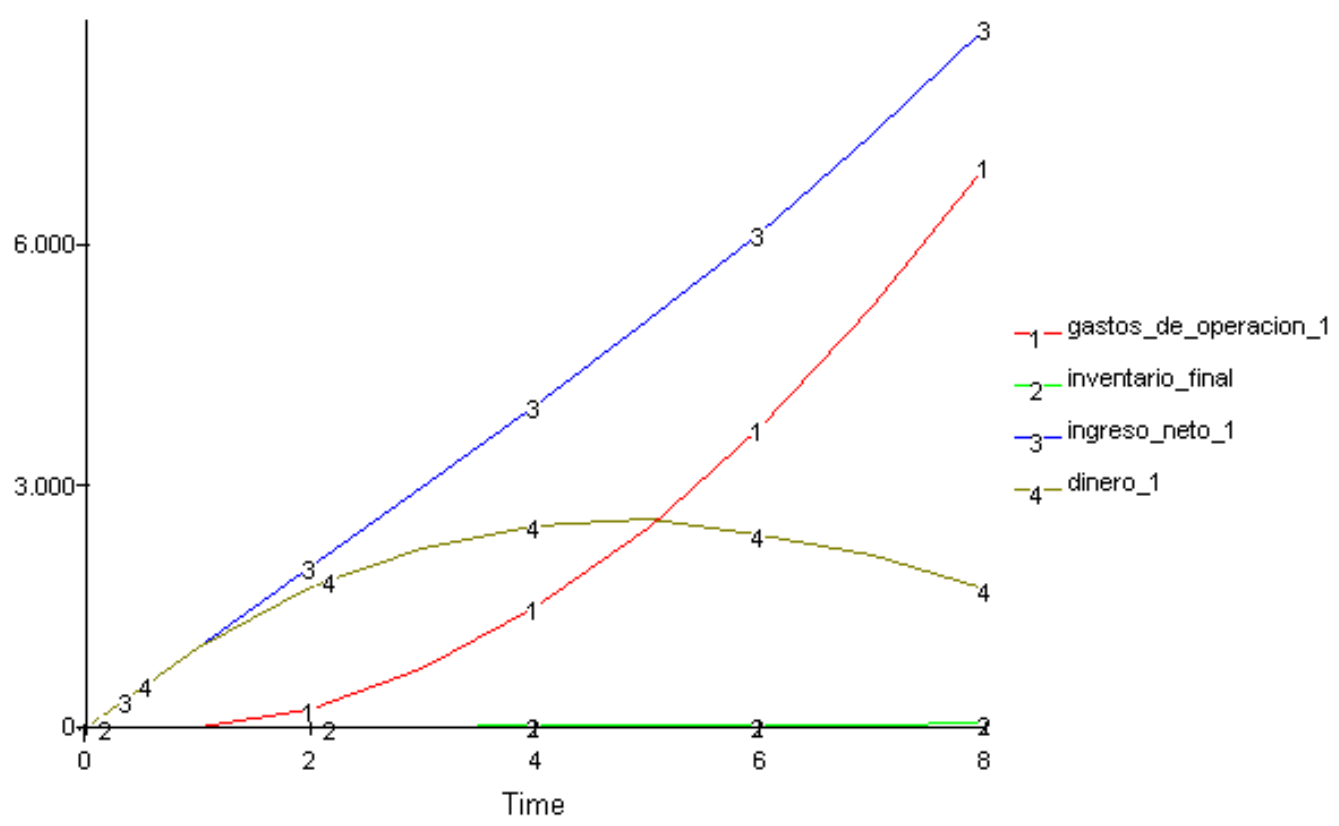

Figura 6. Comportamiento del modelo sin sincronización de las tasas

Figure 6. Model behaviour without rate synchronization

Fuente: elaboración propia.

Tabla 4. Resultados del modelo sin sincronización de las tasas

Table 4. Model Outputs Without Rate Synchronization

\begin{tabular}{ccccc}
\hline Producción /hora & Tasa 1 & Tasa 2 & Tasa 3 & \\
\hline Tiempo & 5 & 3 & 8 & \\
\hline 0 & Gastos de operación & Ingreso neto & $\begin{array}{c}\text { Inventario } \\
\text { final }\end{array}$ & Dinero \\
\hline 1 & $\$-$ & $\$-$ & 0 & $\$-$ \\
2 & $\$-$ & $\$ 1.000,00$ & 8 & $\$ 1.000,00$ \\
3 & $\$ 250,00$ & $\$ 2.011,00$ & 16 & $\$ 1.761,00$ \\
4 & $\$ 750,00$ & $\$ 3.072,00$ & 24 & $\$ 2.322,00$ \\
5 & $\$ 1.500,00$ & $\$ 4.101,00$ & 32 & $\$ 2.601,00$ \\
6 & $\$ 2.500,00$ & $\$ 5.103,00$ & 40 & $\$ 2.603,00$ \\
7 & $\$ 3.750,00$ & $\$ 6.223,00$ & 48 & $\$ 2.473,00$ \\
8 & $\$ 5.250,00$ & $\$ 7.366,00$ & 56 & $\$ 2.116,00$ \\
& $\$ 7.000,00$ & $\$ 8.643,00$ & 64 & $\$ 1.643,00$ \\
\hline
\end{tabular}

Fuente: elaboración propia a partir del diseño de experimentos. 


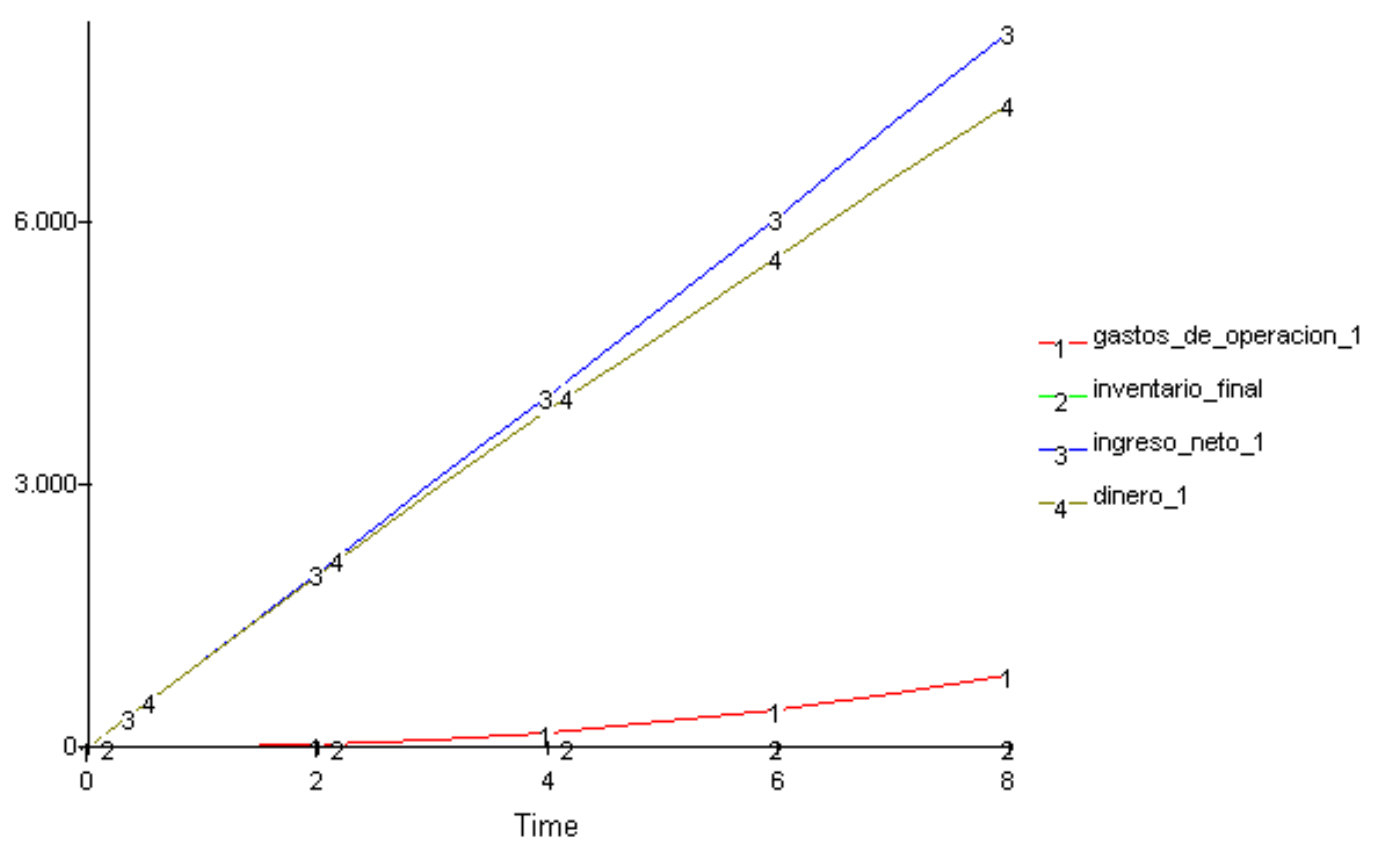

Figura 7. Comportamiento del modelo con sincronización de las tasas

Figure 7. Model Behaviour with rate synchronization

Fuente: elaboración los autores a partir de las simulaciones.

Tabla 5. Resultados del modelo con sincronización de las tasas

Table 5. Model Outputs with Rate Synchronization

\begin{tabular}{ccccc}
\hline $\begin{array}{c}\text { Producción } \\
\text { /hora }\end{array}$ & Tasa 1 & Tasa 2 & Tasa 3 & \\
\hline Tiempo & Gastos de operación & Ingreso neto & $\begin{array}{c}\text { Inventario } \\
\text { final }\end{array}$ & Dinero \\
\hline 0 & $\$-$ & $\$-$ & 0 & $\$-$ \\
1 & $\$-$ & $\$ 1.000,00$ & 3 & $\$ 1.000,00$ \\
2 & $\$ 30,00$ & $\$ 2.010,00$ & 6 & $\$ 1.980,00$ \\
3 & $\$ 90,00$ & $\$ 3.035,00$ & 9 & $\$ 2.945,00$ \\
4 & $\$ 180,00$ & $\$ 4.041,00$ & 12 & $\$ 3.861,00$ \\
5 & $\$ 300,00$ & $\$ 5.060,00$ & 15 & $\$ 4.760,00$ \\
6 & $\$ 450,00$ & $\$ 6.079,00$ & 18 & $\$ 5.629,00$ \\
7 & $\$ 630,00$ & $\$ 7.173,00$ & 21 & $\$ 6.543,00$ \\
8 & $\$ 840,00$ & $\$ 8.217,00$ & 24 & $\$ 7.377,00$ \\
\hline
\end{tabular}




\section{Validación del modelo de simulación}

Muchos autores definen validación como «la correcta conclusión con base en premisas», lo que implica que esté soportada por una verdad objetiva (Sterman, 2000), también se define este concepto como el grado de homomorfismo entre un sistema y un segundo sistema que supuestamente representa (Goto y Takahashi, 2013), otros autores lo consideran el proceso de evaluar el grado de precisión entre el modelo computacional y la representación del mundo real (Ormerod y Rosewell, 2009).

Respecto a esto, se puede afirmar que existen más de 75 técnicas y conceptos de verificación, validación y evaluación de modelos presentadas en la manual de simulación (Banks, 1998). Algunos de estos conceptos incluyen: la validación de modelos conceptuales y la validación operacional (Goto y Takahashi, 2013;Olsen y Raunak, 2013), la primera técnica implica que todos los supuestos sobre el modelo han sido incluidos y la segunda considera que el comportamiento del software sea acorde con lo que se espera del sistema bajo simulación (Olsen y Raunak, 2013).

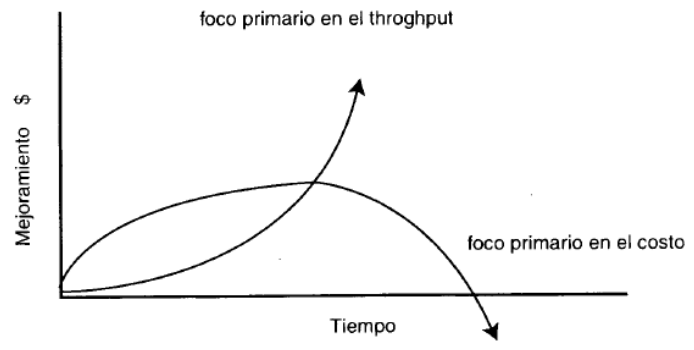

Figura 8. Comportamiento a validar Figure 8. Behavior to validate Fuente: Aguilera (2000).
Con base en esto, Aguilera (2000) presenta en la Fig.8, el comportamiento que debe tener un sistema bajo la TOC, idealmente se espera que el Throughput incremente mientras que los costos disminuyan, de acuerdo con la simulación realizada, se ha evidenciado este comportamiento presente en la Fig.8, al evaluar ambos conceptos se evidencia su relación y equivalencia.

\section{CONCLUSIONES}

La TOC como metodología y herramienta, permite obtener soluciones desde una perspectiva holística del sistema empresarial, estudios realizados basados en evidencia anecdótica sugieren que las empresas que adoptaron esta metodología mejoraron su desempeño, disminuyendo sus inventarios y el tiempo de ciclo de producto, ahora, en comparación con otras metodologías como MRP (Material Requirement Planning) y JIT (Just In Time), rigurosas pruebas estadísticas han probado que la TOC produce mejores resultados evaluando el desempeño corporativo (Nanfang et al., 2008).

La TOC tiene opositores que, aunque son pocos, culpan a dicha metodología de sobre simplificar los problemas complejos; por otro lado, autores alegan que la TOC tiene semejanzas con un libro de recetas, ignorando que para realizar cambios se requiere disciplina matemática y experticia en el balanceo de líneas de trabajo y en optimización, por otra parte, aún continua el debate si la TOC es una teoría o solo un conjunto de procedimiento operativos (Knaggs y Pollard, 2012).

La simulación ofrece beneficios económicos, principalmente este método propuesto facilita y clarifica los conceptos de una metodología que ha sido útil en los ambientes empresariales. 
La simulación también permitió una evaluación más completa y rigurosa de los softwares utilizados, antes de utilizarlos en los procesos de manufactura.

Se puede decir que el método de la TOC es una herramienta que no solo es útil para sincronizar la producción, sino que sirve para mejorar continuamente mientras se trabaja, desde una perspectiva de abstracción de cualquier fenómeno industrial, para su comprensión desde su modelación y simulación.

Como trabajo futuro, se pretende robustecer el modelo, con el fin de ser aplicado a los diferentes fenómenos alusivos a cuellos de botella, desde una perspectiva integral de la simulación basada en agentes y la dinámica de sistemas bajo una plataforma que les integre como por ejemplo AnyLogic.

\section{REFERENCIAS}

Aguilera, C. (2000). Un enfoque gerencial de la teoría de las restricciones. Estudios Gerenciales, 53-69.

Akgün, A. E., Keskin, H., \& Byrne, J. C. (2014). Complex adaptive systems theory and firm product innovativeness. Journal of Engineering and Technology Management, 31(101), 21-42. https://doi.org/10.1016/j.jengtecman.201 3.09.003

Banks, J. (1998). Handbook of Simulation: Principles, Methodology, Advances, Applications, and Practice. (J. Banks, Ed.) (1st ed.). Wiley-Interscience.

Borshchev, A., \& Filippov, A. (2004). From System Dynamics and Discrete Event to Practical Agent Based Modeling: Reasons, Techniques, Tools. In The 22nd International Conference of the System
Dynamics Society (pp. 1-23). Oxford.

Costas, J., Ponte, B., de la Fuente, D., Pino, R., \& Puche, J. (2015). Applying Goldratt's Theory of Constraints to reduce the Bullwhip Effect through agent-based modeling. Expert Systems with Applications, 42(4), 2049-2060. https://doi.org/10.1016/j.eswa.2014.10.02 2

Golddratt, E. (1984). La Meta.

Golmohammadi, D. (2015). A study of scheduling under the theory of constraints. International Journal of Production Economics, 165, 38-50. https://doi.org/10.1016/j.ijpe.2015.03.015

Gonzalez, F., \& Calvachi, N. (2013). Teoría de las restricciones: modelo de gestión gerencial para el crecimiento productivo de las PYMES en Colombia. Caso aplicado a cidma SAS. Colegio Mayor de nuestra señora del Rosario.

Goto, Y., \& Takahashi, S. (2013). Agent-Based Modeling and Simulation Validation by Scenario Analysis. In T. Murata, T. Terano, \& S. Takahashi (Eds.), Agent-Based Approaches in Economic and Social Complex Systems VII (pp. 3-16). Springer Japan. https://doi.org/10.1007/978-4-43154279-7_1

Humann, J., \& Madni, A. M. (2014). Integrated Agent-based modeling and optimization in complex systems analysis. Procedia Computer Science, 28, 818-827. https://doi.org/10.1016/j.procs.2014.03.0 97

Izmailov, A. (2014). If Your Company is Considering the Theory of Constraints. Procedia - Social and Behavioral Sciences, 150,

925-929. 
https://doi.org/10.1016/j.sbspro.2014.09. 103

Kasemset, C. (2011). A Review on Quality Improvement and Theory of Constraints ( TOC ), 327-330.

Knaggs, C., \& Pollard, S. (2012). Applying Theory of Constraints in Administrative Process: An Experiment from the U.S. Government. International Conference on Management Science and Engineering, 2012-2020.

Macal, C. M., \& North, M. J. (2013). Introductory Tutorial: Agent-Based modeling and simulation. Proceedings of the 2013 Winter Simulation Conference, 362-376.

Marín, W., \& Gutierrez, E. (2013). Desarrollo e implementación de un modelo de teoría de restricciones para sincronizar las operaciones en la cadena de suministro. Revista EIA, 67-77.

Murphy, R. (1996). Holistic TOC for maximum profitability. Advance Semiconductor Manufacturing Conference, 242-249.

Nanfang, C., Kaijun, L., \& Tian, W. (2008). Rapid response with TOC methodology.

Olsen, M., \& Raunak, M. (2013). A framework for simulation validation coverage. In Proceedings of the 2013 Winter Simulation Conference (pp. 1569-1580).

Ormerod, P., \& Rosewell, B. (2009). Validation and Verification of Agent-Based Models in the Social Sciences. In F. Squazzoni (Ed.), Epistemological Aspects of Computer Simulation in the Social Sciences (pp. 130140). Springer Berlin Heidelberg. https://doi.org/10.1007/978-3-642-011092_10
Ortiz, F., \& Torres, R. (2008). Comparación del sistema de costos estandar y la teoría de restricciones para el control del flujo de materiales mediante un modelo de simulación. Revista de La Ingeniería Industrial, (1), 1-16.

Quintero, S., Ruiz, W., \& Robledo, J. (2017). Learning in the Regional Innovation Systems: An Agent Based Model. Revista de Administración Y Negocios, 33, 7-20.

Ray, A., Sarkar, B., \& Sanyal, S. (2010). The TOCBased Algorithm for Solving Multiple Constraint Resources. IEEE Transactions on Engineering Management, 57(2), 301-309.

Sargent, R. G. (2015). An introductory tutorial on verification and validation of simulation models. Proceedings of the 2015 Winter Simulation Conference, 1729-1740. Retrieved from http://dl.acm.org/citation.cfm?id=809441

Şimşit, Z. T., Günay, N. S., \& Vayvay, Ö. (2014). Theory of Constraints: A Literature Review. Procedia - Social and Behavioral Sciences, 150(231), 930-936. https://doi.org/10.1016/j.sbspro.2014.0004

Sterman, J. D. (2000). Business Dynamics: Systems Thinking and Modeling for a Complex World. McGraw-Hill/Irwin.

Viveros, L., \& Chew, M. (2013). Entrenamiento para toma de decisiones por medio de simulación y casos de estudio. Revista Internacional de La Educación En Ingenieria, 6(1), 1-7.

Wu, H., \& Tsai, T.-P. (2013). An enhanced model for TOC Supply Chain Replenishment Systems under capacity constraint, 26832688.

Zhang, L. (2009). Using TOC Thinking Process Tools to Improve Safety Performance. 2009 
WRI World Congress on Software

Engineering, 13-17.

https://doi.org/10.1109/WCSE.2009.46 